\title{
LEJOS DEL PODER. HACIA LA IMPLEMENTACIÓN DE UNA LEY DE CUOTAS EN CHILE
}

\author{
Alejandra Zúñiga Fajuri* \\ Daniela Aguilera Bertucci** \\ Andrea Vásquez Bravo ${ }^{* * *}$
}

\begin{abstract}
RESUMEN
El siguiente trabajo pretende justificar, desde el punto de vista del principio de igualdad-tal y como ha sido consagrado en la Constitución chilena-, un sistema de cuotas electorales que ayude a revertir la limitada participación de la mujer en la vida pública. Junto con precisar los conceptos básicos de discriminación inversa y acción afirmativa, se analiza el principio de igualdad y los importantes resultados que los sistemas de cuota utilizados en países como Argentina y Costa Rica ban significado en su consecución y respeto. Finalmente, se reflexiona en torno a la posible aplicación de una ley de cuotas en el sistema binominal chileno.
\end{abstract}

\section{DISCRIMINACIÓN - ACCIÓN POSITIVA- LEY DE CUOTAS}

Far away from power. Towards the implementation of a quota system law in Chile

\section{ABSTRACT}

This essay intends to justify, from the point of view of equality - as it has been stated in the Chilean Constitution - a system of electoral quotas that helps to reverse the limited participation of women in public life. The paper describes the basic concepts of discrimination and affirmative action. In addition, it analyzes the principle of equality and the positive results of quotas systems in countries such as Argentina and Costa Rica. Finally, it studies the possible application of a quotas law in the Chilean electoral system.

\section{DISCRIMINATION - POSITIVE ACTION - QUOTA LAW}

* Abogada, Doctora en Derecho, Profesora e investigadora de la Escuela de Derecho de la Universidad Católica de Temuco, Chile, azunigaf@uctemuco.cl

** Licenciada en Ciencias Jurídicas y Sociales de la Universidad Católica de Temuco, Chile, daguilbe@alu.uct.cl

*** Licenciada en Ciencias Jurídicas y Sociales de la Universidad Católica de Temuco, Chile, avasquezquez@alu.uct.cl

Artículo recibido el 10 de agosto de 2007 y aceptado para su publicación por el Comité Editorial el 22 de octubre de 2007. 


\section{INTRODUCCIÓN}

$\mathrm{L}$ as mujeres están muy lejos del poder en Chile. Su nivel de participación en todas las instituciones sociales básicas -excepto en la institución de la familia- es mínimo. ¿Cuáles son las causas de este fenómeno? ¿Se debe a que las chilenas están menos interesadas que las mujeres del resto del mundo en participar de las cuotas de poder que generan los cargos de mayor responsabilidad y mejor remuneración? ¿Será acaso que las chilenas carecen de los méritos necesarios - de las capacidades y habilidades- que se requieren para participar en la vida pública?

Se suele sostener que son los factores socioeconómicos, como la tasa de participación económica de las mujeres (que en Chile es especialmente baja a nivel mundial pues se empina poco más allá del $30 \%$ ), la disparidad en los niveles de educación y las remuneraciones, lo que condiciona su acceso igualitario a cargos de representación política. Asimismo, dimensiones culturales relacionadas con determinadas percepciones de lo que constituye el rol de la mujer y el hombre en la sociedad tendrían también una consecuencia similar. Con todo, como demuestran diversos estudios, ${ }^{1}$ los efectos de los factores culturales o socioeconómicos son más bien indirectos y de largo plazo, de modo que son elementos estrictamente políticos los que determinan las oportunidades y las barreras que enfrentan las mujeres para acceder a los cargos de elección popular. ${ }^{2}$

La ley italiana $\mathrm{N}^{\circ}$ 125/1991 advierte que las discriminaciones de las que las mujeres son víctimas en la esfera profesional son el fruto de una contaminación de injusticia en su situación en la esfera privada (desigual repartición de los trabajos domésticos entre cónyuges). Las mujeres son mayoritariamente tratadas en la esfera de la producción como trabajadoras sin un proyecto porque ya están investidas en la esfera privada de un rol social fundamental, el de ser madres y guardianas del hogar. Consecuentemente el objetivo perseguido por las acciones positivas no es sólo restablecer las fronteras entre la esfera privada y la profesional sino igualmente redibujar los roles. ${ }^{3}$

El último Informe de competitividad del Foro Económico Mundial 2006-2007 da cuenta de la desigual condición de la mujer chilena en el acceso a cargos de alta responsabilidad, colocándose al país en el lugar 110 de 125 economías de todo el mundo. En este contexto, es necesario analizar uno de los más importantes mecanismos de equiparación de oportunidades a favor de minorías históricamente excluidas y relacionado con las acciones positivas, cual es, las llamadas "leyes de cuotas", que han sido adoptados progresivamente en todo el mundo con el fin de mejorar la participación femenina en

${ }^{1}$ Unión Europea. Impacto diferencial de los sistemas electorales en la representación politica femenina. Dirección General de Estudios. Serie: Derechos de la mujer. 1997; IDEA (Institute for Democracy and Electoral Assistance), Women in Parliament: Beyond Numbers. A Revised Edition, IDEA, Estocolmo, 2005.

${ }^{2}$ Ríos, M. y Villar, A., Cuotas de género: democracia y representación, IDEA, FLACSO-Chile, Santiago, Chile, 2006, p. 14.

${ }^{3}$ Citada por Kemelmajer, A., "Las acciones positivas" en Miguel Carbonell (compilador), El principio constitucional de igualdad. Lecturas de introducción. Comisión Nacional de los Derechos Humanos de México, México, 2003, p. 241. 
la política y, en general, en la vida pública. Desde 1991, once países en Latinoamérica han incorporado leyes de cuotas u cuotas de partidos a sus ordenamientos jurídicos y cincuenta lo han hecho en todo el mundo. En Chile, donde se ha iniciado la discusión respecto de la viabilidad, justicia y necesidad de implementar cuotas electorales, se requiere de un análisis de los aspectos controversiales que tales mecanismos plantean así como de su justificación a partir del principio de igualdad.

Todo estudio que se concentre en este tipo de sistema de equiparación de oportunidades debe necesariamente hacerse cargo de los cuestionamientos que surgen en relación con la legitimidad de establecer condiciones preferenciales en favor de las mujeres para permitirles alcanzar una mayor participación en los cargos de poder. En la misma línea es crucial preguntarse, desde la reflexión en torno a los sistemas de cuotas actualmente vigentes, por su verdadera aptitud para lograr aquellos cambios que se esperan con su implementación. El examen de la necesidad de este tipo de leyes requiere evaluar su idoneidad, efectividad y capacidad para generar mayores niveles de igualdad social.

El siguiente trabajo se ocupa, primeramente, de describir los grados de participación de las mujeres en Chile y el mundo para, a continuación, explicar los mecanismos de discriminación inversa que se han utilizado desde la década del 70 para superar la subrepresentación de ciertos grupos sociales históricamente postergados. Luego se analizan el principio de igualdad y su relación con las medidas de discriminación y las leyes de cuotas electorales, ¿son incompatibles? ¿Es cierto que los mecanismos de cuotas nos exigen violar el derecho al trato igual de algunas personas? Se pasa revista, a continuación, a los modelos de cuotas electorales aplicados en Costa Rica y Argentina, para terminar con el análisis de las objeciones sobre las posibilidades de aplicar una ley de cuotas en Chile con el actual sistema binominal.

\section{La PARTiCipación de LA MUJER EN Chile y EL MUNDO}

Actualmente la media mundial de mujeres parlamentarias es del 16,9\%. En un contexto mundial los países nórdicos se colocan en el primer lugar con aproximadamente 40\% de mujeres en sus parlamentos, los países de "las Américas" tienen un 20,7\%, los países de la OSCE (Organization for Security and Cooperation in Europe) poseen un $17 \%$ de parlamentarias, África subsahariana tiene un 16,6\%, Asia cuenta con un 16,1, los países de Asia-Pacífico tienen un 13,8, Chile sólo un 12,6 y los países árabes con un $7,7 \% .^{4}$

Si bien la inserción de la mujer al mercado laboral ha aumentado considerablemente durante la última década en Chile, lo cierto es que dicho incremento es menor si se le compara con los países del resto de la región. En efecto, el promedio de América Latina es cercano al $44 \%$, mientras que en Chile ha variado desde un $28 \%$ en 1992 a un $36 \%$ en

\footnotetext{
${ }^{4}$ Ríos y Villar, op. cit., p. 11.
} 
el año 2002..$^{5}$ En cuanto a la participación política, las tasas han variado en los últimos años. En el año 2000 sólo un 29, $2 \%$ de mujeres estaba a cargo de ministerios, subsecretarías, intendencias y gobernaciones, cifra que durante los años siguientes descendió paulatinamente, alcanzando en el año 2005 un porcentaje aproximado del 20,5\%. Si bien con el gobierno de Bachelet la cantidad de mujeres que ejercen cargos de confianza ha aumentado notablemente, logrando una tasa del $41,2 \%$, es razonable predecir que dicha política paritaria desaparecerá junto con el fin de su gobierno. ${ }^{6,7}$

En este contexto, como se adelantó, destaca el lamentable porcentaje de participación de las chilenas en el Parlamento. Si bien según el último censo del año 2002 en Chile la población femenina corresponde al 50,7\% (cifra que aumenta al analizar el padrón electoral, el cual está compuesto en un $52,4 \%$ por votantes mujeres) el Congreso Nacional sólo posee un 12,6\% de mujeres, es decir, sólo 2 de los 38 senadores y 18 de los 120 diputados. Esto parece reproducir, por lo demás, la escasa presencia de mujeres en las candidaturas parlamentarias pues, si bien el porcentaje varía dependiendo del partido político, en ninguno de ellos éstas han superado el $28 \%$ del total de candidatos. ${ }^{8}$ Así, de continuar los actuales niveles de crecimiento en la incorporación de la mujer en la esfera pública, las chilenas debieran esperar al menos 40 años para que los promedios de representación alcancen niveles cercanos al $40 \% .{ }^{9}$ Es por ello que el mecanismo de las leyes de cuotas, como medidas de discriminación positiva, resulta atractivo a quienes consideran que la espera ya ha sido demasiado larga.

\section{MEDIDAS DE DISCRIMINACIÓN POSITIVA}

La discriminación inversa tuvo su origen en Estados Unidos en los años setenta, adquiriendo gran notoriedad a través de los casos De Funnis y Bakke, ambos vinculados al favorecimiento de minorías raciales con escasa participación en niveles universitarios. El primero de ellos se origina en 1971 por un estudiante de origen judío que fue rechazado por la Facultad de Derecho de la Universidad de Washington debido a su política de

${ }^{5}$ Cabe destacar que, además, en Chile esta tasa varía de acuerdo a los distintos quintiles de ingreso, por lo que la población femenina económicamente activa es mucho menor en los sectores pobres, llegando a un $24,8 \%$ en el primer quintil de ingreso, muy por debajo del quinto quintil, donde la participación alcanzó un 52,1\% en el año 2000. Servicio Nacional de la Mujer, Informe de seguimiento de la aplicación de la Plataforma de Acción de Beijing presentado por el Gobierno de Chile ante la División para el Adelanto de la Mujer de Naciones Unidas, Santiago, 2004, p. 12.

${ }^{6}$ Servicio Nacional de la Mujer, información estadística, disponible en: http://www.sernam.cl/basemujer/index.htm.

${ }^{7}$ Bórquez, R.; Ordaz, C., "Participación de las mujeres en organismos internacionales de protección de derechos humanos y en los sistemas judiciales sudamericanos”, en Serie de Documentos técnicos y jurídicos, Corporación del desarrollo de la mujer, Domos, año 2, Santiago, 2005, pp. 77-80.

${ }^{8}$ Ríos y Villar, op. cit., p. 26.

${ }^{9}$ Corporación La Morada, Mujer-sujeto, mujeres- sujetadas, Informe sobre la situación de los derechos bumanos de las mujeres en Chile. Derechos civiles y políticos, Santiago, 2004, p. 56. 
discriminación inversa, la que establecía un sistema de selección que otorgaba puntos extras para minorías raciales desfavorecidas. Si bien el caso no se resolvió judicialmente, dio lugar a un interesante debate doctrinal que estimó, en general, que la universidad no vulneraba los derechos fundamentales que concedía la Constitución estadounidense. Dworkin justificó la decisión de la universidad sosteniendo que "los criterios raciales no son necesariamente los estándares correctos para decidir qué aspirantes deben ser aceptados por las facultades, pero tampoco lo son los criterios intelectuales, ni -a decir verdad- ningún otro conjunto de criterios (...) el programa se justifica si sirve a una política adecuada, que respete el derecho de todos los miembros de la comunidad a ser tratados como iguales" que es lo que, en su concepto, había hecho la Facultad de Derecho de la Universidad de Washington. ${ }^{10}$

Por su parte, el caso Bakke, conocido por la Suprema Corte de Estados Unidos en 1978, decidió la situación de un estudiante blanco rechazado por la Facultad de Medicina de la Universidad de California, la cual reservaba un número determinado de plazas para minorías desfavorecidas desde el punto de vista educativo y económico. Tal reserva fue estimada inconstitucional por la Corte Suprema estadounidense. Dworkin opinó, sobre este caso, que si bien Allan Bakke no es el responsable de que la justicia racial sea una necesidad contingente, esto no significa que tenga el derecho a impedir el uso de la medida más efectiva que existe para garantizarla. ${ }^{11} \mathrm{~A}$ pesar del fallo de la Corte, lo importante del caso Bakke es que abrió la puerta a las medidas de discriminación positiva que consideran la raza como un elemento o rasgo relevante, en ciertos casos, para fundamentar el test de razonabilidad que se exige al momento de aplicar el principio de igualdad.

Desde entonces, la Corte Suprema norteamericana ha fallando a favor o en contra de las medidas de discriminación positiva dependiendo de su composición y del tipo de medida de que se trate. En 1989, en City of Richmond c/J.A.Croson, declaró inconstitucional un programa municipal, llamado "plan Richmond”, que obligaba a las empresas contratistas del Estado a subcontratar, por lo menos, un treinta por ciento de cada contrato con empresas comerciales que pertenecían a determinados grupos raciales o étnicos. En cambio, en 1995, en el caso Adarand Constructors Inc c/ Peña, decidió que cualquier programa federal de acción afirmativa debe ser examinado bajo el método del llamado "examen exigente" que supone que si la clasificación se hace según un criterio sospechoso (por ejemplo, la raza), la clasificación es constitucional sólo si está estrechamente diseñada para promover un interés gubernamental prioritario. ${ }^{12}$

${ }^{10}$ Para este autor, la "Cláusula de igual protección" -que no tiene un contenido único e invariable-efectivamente da rango constitucional al derecho a ser tratado como igual, pero ese derecho no puede servir de argumento para quien pretenda sostener que dicha cláusula convierte en ilegales todo tipo de clasificaciones raciales. Dworkin, R., op. cit., pp. 334-347.

${ }^{11}$ Dworkin, R., A matter of principle, Harvard University Press, 1985, pp. 302-303.

${ }^{12}$ Ruiz Miguel ha hecho una traducción de la construcción realizada por la Corte Suprema estadounidense. Ésta se basa en los diferentes test utilizados por dicha Corte en el juzgamiento de alegaciones de violación de la cláusula de la igual protección de las leyes garantizada por la Enmienda XIV. De este modo, 
En Europa, por su parte, el caso más comentado ha sido el caso Kalanke cuya sentencia de 17 de octubre de 1995 fue la primera en la que el Tribunal de Justicia de las Comunidades Europeas (TJCE) interpretó la Directiva 76/207, artículo 2, en sus apartados 1 y 4 que disponían un régimen de cuotas establecido en favor de las mujeres. ${ }^{13}$ En Bremen (Alemania), la ley relativa a la igualdad de trato entre sexos en la función pública disponía que en la selección de candidatos para el ingreso y promoción en un determinado puesto tendrían preferencia las mujeres "con igual capacitación” que los hombres y siempre que se encontraran infrarrepresentadas en dicho sector (lo que se considera ocurre cuando no cubren al menos la mitad de los puestos). Bremen llevó a cabo una selección para cubrir un puesto de promoción de jefe de sección en el servicio de parques y jardines y, tras finalizar el proceso, dos candidatos que cumplían el perfil del puesto requerido fueron clasificados, el Sr. Kalanke y la Sra. Glissmann. En un primer momento se propuso el ascenso del Sr. Kalanke, pero el Comité de conciliación consideró que ambos candidatos tenían idéntica capacitación y que, por lo tanto, debía darse preferencia a la Sra. Glissmann. Ante esta situación, el Sr. Kalanke alegó que poseía una capacitación mayor y que el régimen de cuotas establecido por la Ley del Estado de Bremen era contrario a la Ley Fundamental (al principio de igualdad) y al artículo 611 del Código Civil alemán.

El Tribunal de Justicia de las Comunidades Europeas (TJCE) resolvió que el apartado 4 del artículo 2 de la Directiva 76/207, que tiene como objetivo aplicar en los Estados miembros el principio de igualdad de trato entre ambos sexos en el empleo (incluida la promoción), constituía una excepción al principio de igualdad de trato entre hombres

la jurisprudencia puede sistematizarse en tres tipos diferentes de test: "(a) un test estricto -denominado Strict Scrutiny-, para las distinciones normativas que afectan derechos fundamentales o son 'sospechosas' (esto es, utilizan la raza o, con menos rotundidad, la filiación ilegítima), conforme al cual los fines de la norma o medida deben ser 'perentorios' (compelling) y la distinción 'necesaria' (necessary) para la realización de tales fines, (b) un test mínimo -denominado Minimum Rationality Requirement o Rational Basis Test-, utilizado en principio para la generalidad de las distinciones normativas, que exige únicamente que los fines sean 'lícitos' (legitimate) en el marco de alguna concepción del interés general y que la distinción esté 'racionalmente relacionada' (rationally related) con ellos; y, en fin, (c) un test intermedio -llamado Intermediate Review-, que se ha ido abriendo paso entre los dos anteriores para las distinciones que utilizan categorías 'sensibles' (aunque no 'sospechosas'), como el sexo, la discapacidad o la edad, y por el que se exige que el fin de la norma o medida sea 'importante' (important) y que la distinción esté 'sustancialmente relacionada' (substantially related) con tal fin”. Ruiz Miguel, A. "La Igualdad en la Jurisprudencia del Tribunal Constitucional”, en $E l$ Principio de Igualdad, Luis García San Miguel (ed.), Universidad de Alcalá de Henares-Dykinson, Madrid, 2000, p. 177.

${ }^{13}$ La Directiva 76/207 pretende garantizar la aplicación en los Estados miembros del principio de igualdad de trato entre hombres y mujeres en lo que se refiere al acceso al empleo y la promoción profesional. El apartado 1 del artículo 2 de la Directiva establece que el principio de igualdad de trato supone "la ausencia de toda discriminación por razón de sexo, bien sea directa o indirectamente, en lo que se refiere, en particular, al estado matrimonial o familiar”. El apartado 4 del mismo artículo dispone que la Directiva no obstará a "las medidas encaminadas a promover la igualdad de oportunidades entre hombres y mujeres, en particular para corregir las desigualdades de hecho que afecten a las oportunidades de las mujeres”. Este apartado tiene como finalidad autorizar medidas que, aunque sean discriminatorias en apariencia, están destinadas a eliminar o a reducir las desigualdades de hecho que pudieran existir entre hombres y mujeres. 
y mujeres, de modo que debía ser aplicado restrictivamente. Por lo mismo, decidió que una normativa nacional que "garantiza la preferencia absoluta e incondicional de las mujeres en un nombramiento o promoción sobrepasa lo que debe ser el fomento de la igualdad de trato". ${ }^{14}$ Este caso fue sometido a revisión en el caso Marschall, que resolvió que en el evento de que candidatos de ambos sexos presenten igual capacitación, desde el punto de vista de su aptitud, competencia y prestaciones profesionales, es legítimo conceder preferencia en la promoción a las candidatas femeninas en aquellos sectores de actividad de la administración que, en el nivel del puesto de que se trate, tengan un menor número de mujeres que de hombres. ${ }^{15}$

Habiendo pasado revista brevemente a los casos citados, podemos ahora conceptualizar la discriminación positiva o inversa, junto con Alfonso Ruiz Miguel, como una clase específica de acción afirmativa que se caracteriza por dos elementos distintivos: primero, la medida diferenciadora correctora es discriminatoria y no simplemente desigualitaria, en el sentido que se refiere a un tipo muy especial de desigualdad, por rasgos como el sexo, la raza o similares, rasgos que tienen la cualidad de ser transparentes e inmodificables para los individuos que los portan y son considerados por la sociedad tendencial y generalmente de forma negativa. Segundo, la discriminación inversa se produce en una situación de especial escasez, como suele ocurrir con los puestos de trabajo o plazas universitarias, lo que provoca que se considere que el beneficio a ciertas personas tiene como forzosa contrapartida un claro y visible perjuicio de otras. ${ }^{16}$

\section{EL PRINCIPIO DE IGUALDAD Y LAS CUOTAS}

La igualdad tiene que ver con normas sociales pues no son los hechos los que definen la relación entre los seres humanos, sino la valoración normativa que se da a esos hechos (el significado y consecuencias que les atribuyen). De este modo, la igualdad es un principio, no la descripción de una realidad -es un concepto normativo, no descriptivo-, pues no se ocupa de cómo son las cosas, sino de cómo "deberían ser". La igualdad, luego, es una metanorma, es decir, un principio cuyo sentido y función es metanormativo respecto de las normas de la sociedad, por lo que se le concibe como un mensaje prescriptivo dirigido a ellas, como una exigencia genérica frente a la estructura, contenido y efectos de esas normas sociales. ${ }^{17}$

\footnotetext{
${ }^{14}$ Es evidente, como sostiene Ruiz Miguel, que en el caso Kalanke la preferencia de la mujer no era incondicional ni absoluta pues, precisamente en ese caso, se requería igualdad de méritos con los postulantes hombres de modo que un sistema de cuotas como el que proponía el tribunal -un sistema de puntos, por ejemplo- habría tenido el mismo efecto que finalmente tuvo la regulación de Bremen. Para un análisis detallado del caso véase Ruiz Miguel, A., "La discriminación inversa y el caso Kalanke”, Doxa No 19, 1996.

${ }^{15}$ Sentencia del caso Marschall del 11 de noviembre de 1997, considerando 24.

${ }^{16}$ Ruiz Miguel, A., "Discriminación inversa e igualdad”, en A. Valcárcel (compiladora), El concepto de igualdad, Editorial Pablo Iglesias, Madrid, 1994, p. 80.

${ }^{17}$ Laporta, F., "El principio de igualdad. Introducción a su análisis”, Sistema, Revista de Ciencias Sociales, Madrid, 1985, pp. 3 y ss.
} 
¿Cómo podría definirse el principio de igualdad? Siguiendo a Dworkin, el principio de igualdad implica que el gobierno debe "tratar a todas las personas con igual consideración y respeto", esto es, como seres humanos capaces de sufrimiento y de frustración y como individuos facultados para llegar a concepciones racionales de cómo han de vivir su vida, y de actuar en conformidad a ellas. ${ }^{18}$ De este modo, tratar a las personas como iguales exige al Estado no distribuir bienes u oportunidades de manera desigual, basándose en que algunos sujetos tienen "derecho a más" porque son dignos de mayor consideración. La cuestión crucial dentro de un Estado es, prosigue Dworkin, la de cuáles son las desigualdades en bienes, oportunidades y libertades que se permiten y por qué razones. Ahora bien, expresar el principio no ofrece ninguna dificultad, pero ponerse de acuerdo sobre su significado preciso requiere un esfuerzo teórico importante. Lo relevante es comparar este principio abstracto con las normas que deben satisfacerlo, es decir, verificar bajo qué condiciones una norma cumple el principio de igualdad.

Por cierto que todo principio moral o jurídico, y toda norma también, se enfrentan con la realidad e intenta modificarla o legitimarla. Para Laporta, la realidad a la que se enfrenta el principio de igualdad es el amplio espectro de rasgos, características, habilidades, talentos y circunstancias que tienen que ver con los seres humanos, esto es, aquello que hace semejantes y diferentes a las personas. El principio de igualdad actúa precisamente sobre esta realidad considerando en algunas oportunidades que dichas semejanzas son relevantes y, en otros casos, que no lo son. Así, es un principio que despliega a su vez un abanico de principios de segundo orden y de normas concretas que se ocupan de catalogar los rasgos que diferencian a los seres humanos en función de su relevancia o irrelevancia. De esos principios y normas los que más directa e intuitivamente parecen expresar la idea de igualdad son aquellos que determinan la cancelación de ciertos rasgos distintivos como razones relevantes para diferenciar en el tratamiento normativo. Ejemplo típico de esto es la interdicción de rasgos como la raza, el sexo, la clase social, las convicciones religiosas o políticas, etc. ${ }^{19}$

Igualdad es, de modo inmediato, no discriminación y no discriminación es simplemente la cancelación de ciertos rasgos como razones relevantes para la diferenciación normativa. Ahora, el principio de igualdad, correctamente entendido, contiene dos subprincipios que, siguiendo la clásica máxima de Aristóteles que nos pide "tratar igual lo que es igual, y diferente lo que es diferente", nos permiten hacer la distinción tradicional entre igualdad por equiparación e igualdad por diferenciación. ${ }^{20}$ La primera opera cuando se considera que las diferencias fácticas que concurren son irrelevantes y deben ser descartadas y la segunda funciona cuando se considera que las diferencias fácticas son relevantes y deben ser consideradas para atribuir determinadas consecuencias normativas.

${ }^{18}$ Dworkin, R., Los Derechos en serio, Ariel, Barcelona, 1999, pp. 388-389.

${ }^{19}$ Laporta, op. cit., pp. 14 y ss.

${ }^{20}$ Laporta, op. cit., pp. 14-15; Peces-Barba, G., Los Valores Superiores, Tecnos. Madrid, 1983, pp. 152-156. 
De este modo, estaremos en presencia de una aplicación de las reglas de la "igualdad por equiparación” cuando consideramos que la raza o el sexo de las personas son irrelevantes para atribuir el derecho a sufragio. Al contrario, nos moveremos en el modelo de "igualdad por diferenciación" cuando mantenemos que la raza o el sexo son, esta vez, relevantes a la hora de establecer cuotas que garanticen su igual presencia en la universidad o en cargos públicos de importancia. Se destacan como ejemplos situaciones en que el mismo rasgo es considerado en un caso relevante y en el otro irrelevante para poner de manifiesto que, al final de cuentas, todas las discusiones sobre la igualdad terminan en la disputa sobre la importancia o relevancia de los rasgos a considerar en determinadas circunstancias.

El principio de igualdad pretende señalar con nitidez la frontera entre la relevancia y la irrelevancia de los rasgos, pues precisamente porque prescribe que frente a rasgos irrelevantes no cabe hacer diferenciación en las consecuencias normativas, necesariamente determina también que, cuando en las condiciones de aplicación de la norma aparezca un rasgo relevante diferenciador, mantener el tratamiento normativo igual, es decir, no diferenciar, sería incorrecto. Podría decirse, luego, que tan contrario al principio es proponer diferentes consecuencias normativas en base a rasgos "irrelevantes", como proponer la misma consecuencia normativa para dos supuestos ignorando la presencia de rasgos "relevantes" en uno de ellos. "Tan incorrecto es no conceder una medalla olímpica a un atleta negro por ser negro, que no concedérsela a un atleta blanco aunque sea el vencedor". 21

Existe una presunción a favor del tratamiento igual y, por ende, de la interdicción de la discriminación, es decir, el modelo de igualdad por equiparación. Por esta razón, las discusiones en torno a la aplicación del principio de igualdad se reducen, desde el punto de vista de su importancia, a la consideración y justificación de la diferencia o la desigualdad. Hay una diferencia cualitativa entre tratamiento igual y tratamiento desigual, por lo que, siguiendo a Alexy, si la desigualdad es diferenciación arbitraria cuando no es posible encontrar una razón que la justifique, habiendo razón suficiente para la diferenciación, debe darse un trato desigual. ${ }^{22}$ Luego, la igualdad por diferenciación debe probarse pues, a falta de ello, opera la primera regla, es decir, el principio básico de no discriminación. Las medidas de discriminación inversa o positiva se relacionan, entonces, con la igualdad por diferenciación que obliga a tratar desigualmente a los desiguales cuando se ha determinado, de acuerdo a un juicio de relevancia, que ciertos rasgos o circunstancias fácticas son relevantes.

${ }^{21}$ Laporta, op. cit., p. 15.

${ }^{22}$ El principio de igualdad podría formularse, en definitiva y siguiendo a Alexy, en la siguiente regla de justicia que se divide en dos subprincipios que operan uno tras de otro: a) "Si no hay ninguna razón suficiente para la permisión de un tratamiento desigual, entonces está ordenado un tratamiento igual"; y b) "Si hay una razón suficiente para ordenar un tratamiento desigual, entonces está ordenado un tratamiento desigual”. Alexy, R., Teoría de los Derechos Fundamentales, Centro de Estudios Políticos y Constitucionales, Madrid, 2002, p. 395. 
La connotación desfavorable que tiene el término discriminación influye en la valoración negativa que se hace de la discriminación inversa. Siguiendo a Nino, "La carga emotiva de las expresiones lingüísticas perjudica su significado cognoscitivo, favoreciendo su vaguedad, puesto que si una palabra funciona como una condecoración o como un estigma, la gente va manipulando arbitrariamente su significado para aplicarlo a los fenómenos que acepta o repudia”. ${ }^{23}$ Al no utilizar el término discriminación en su sentido descriptivo, y al asimilarlo tradicionalmente con distinciones de carácter arbitrarias o injustas, se genera un prejuicio en contra de las medidas de discriminación inversa o positiva, que resulta difícil de superar. Con todo, la utilización del término discriminación inversa se debe, probablemente, a que no hay alternativa que describa mejor la naturaleza de la medida, que alude a la inversión de una discriminación precedente mediante una discriminación de signo opuesto. ${ }^{24}$ Lo importante, entonces, es tener en cuenta la carga emotiva que tiene el concepto "discriminación” al analizar los mecanismos de discriminación inversa. Se debe ser cuidadoso de no caer, como expresa Ruiz Miguel, en la tentación inmediata de resolver verbalmente el problema de su justificación. ${ }^{25}$

Ahora, entre las objeciones que se hacen a las acciones de discriminación positiva destaca aquella que sostiene que se trata de una medida contraria al principio de igualdad pues éste proscribiría toda diferencia basada en criterios no relevantes, como lo son la raza o el sexo. Sin embargo, como vimos, precisamente en función de este principio es que se articulan medidas como las leyes de cuotas pues la discriminación positiva se relaciona con la igualdad por diferenciación. En esta línea, John Rawls argumentó que "para instaurar la igualdad entre hombres y mujeres en el reparto del trabajo en la sociedad, en la preservación de su cultura y en la reproducción de sí misma a lo largo del tiempo, se necesitan cláusulas especiales en el derecho familiar (y sin duda en más sitios), de tal forma que la carga de dar a luz, de criar y educar a los hijos no recaiga más únicamente en las mujeres, pues ello socavaría su equitativa igualdad de oportunidades". ${ }^{26}$

El principio de igualdad, entonces, exige al Estado corregir las desigualdades injustas mediante -si es necesario- otras desigualdades de naturaleza compensatoria. Rawls, al analizar en Teoría de la Justicia el "principio de diferencia" -incluido en su segundo principio de justicia- sostiene que "con objeto de tratar igualmente a todas las personas y de proporcionar una auténtica igualdad de oportunidades, la sociedad tendrá que dar mayor atención a quienes tienen menos dotes naturales y a quienes han nacido en las posiciones sociales menos favorables. La idea es compensar las desventajas contingentes

${ }^{23}$ Nino, C.S., Introducción al análisis del derecho, Ariel Derecho, Barcelona, 2003, p. 269.

${ }^{24}$ Ruiz Miguel, A., "Discriminación inversa e igualdad”, cit., p. 81. M. Ángeles Barrère señala que existe un uso ideológico del lenguaje y que por tanto, desde una perspectiva teórica, el empleo de ambas expresiones resulta improcedente. "Igualdad y discriminación positiva: Un esbozo de análisis teórico conceptual”, en http://www.uv.es/CEFD/9/barrere1.pdf.

25 Ruiz Miguel, A., "La discriminación inversa y el caso Kalanke”, op. cit., p. 127.

${ }^{26}$ Rawls, J., La justicia como equidad. Una reformulación. Paidós, Barcelona, 2002, p. 34. 
en dirección hacia la igualdad". ${ }^{27}$ Asimismo Norberto Bobbio sostiene que "no resulta superfluo reclamar la atención sobre el hecho de que, precisamente con el objeto de situar individuos desiguales por nacimiento en las mismas condiciones de partida, puede ser necesario favorecer a los más desposeídos y desfavorecer a los más acomodados, es decir, introducir artificialmente, o bien imperativamente, discriminaciones de otro modo no existentes. Es así como una desigualdad se convierte en instrumento de igualdad, por el simple motivo de que corrige una desigualdad precedente". ${ }^{28}$

El principio de igualdad, concebido de la forma antes descrita, no sólo permite sino que hace imperativo el establecimiento de medidas discriminatorias justificadas que tengan como finalidad una mayor equidad. Por ello, la prohibición de discriminar alcanza sólo a la discriminación arbitraria o injusta, la cual presenta características que no concurren de manera alguna en la discriminación inversa. Alfonso Ruiz Miguel señala que en la discriminación injusta existe, primero, una motivación social minusvaloradora, despectiva o estigmatizante, que considera a las personas o grupo discriminado como inferiores o incapaces de realizar ciertas tareas; en segundo lugar, posee una finalidad de desigualdad injusta, que tiene como propósito mantener y perpetuar la inferioridad de los grupos discriminados; tercero, posee un contenido que implica la negación o restricción de derechos básicos, incluso a través de medios jurídicos; y, por último, tiene un resultado o efecto social que, en los hechos, tiende a mantener o consolidar la subordinación, marginación u opresión del grupo discriminado respecto del grupo que ejerce la discriminación. ${ }^{29}$

La discriminación inversa no presenta tales características pues a) no existe motivación social despectiva, porque no entraña ningún tipo de menosprecio por los hombres; b) tampoco hay una finalidad de desigualdad injusta, toda vez que el objetivo de ella es precisamente conseguir una mayor igualdad entre grupos injustamente discriminados con anterioridad, y c) el objeto de la discriminación inversa no afecta nunca a los derechos básicos que, como el voto, la libertad de reunión o el derecho a defensa, no son sometibles a balances con fines sociales genéricos, sino que sólo alcanza a la distribución de derechos o bienes sociales en los que, en diferentes contextos, se aceptan restricciones legales por finalidades de interés social. ${ }^{30}$

\footnotetext{
${ }^{27}$ Ralws, J., A Theory of Justice, Oxford University Press, Londres, pp. 100-1.

${ }^{28}$ Bobbio, N., Igualdad y libertad, Editorial Paidós, Barcelona, 1993, p. 79.

${ }^{29}$ Las características identificadas por el autor están o han estado presentes en la discriminación sufrida históricamente por la mujer. Así, la preferencia laboral por los hombres ante las cargas familiares de las mujeres, aunque puede presentarse externamente como meramente utilitaria por parte del empresario, depende finalmente de un criterio social minusvalorador, residuo de una larga historia que la relega a la esfera privada, que es, a fin de cuentas, la menos importante. En cuanto a la restricción de derechos básicos, lo cierto es que no está tan lejana la exclusión de la mujer del derecho a voto o su calificación de jurídicamente incapaz y sin plena disposición sobre su propia persona y bienes frente al marido. Ruiz Miguel, A., "La discriminación inversa y el caso Kalanke", op. cit., p. 129.

${ }^{30}$ Ibid., p. 130.
} 
Por lo tanto, no es posible calificar a la discriminación inversa como contraria al principio de igualdad, ya que se diferencia de la discriminación injusta tanto en su finalidad como en sus características, toda vez que su objetivo es, precisamente, corregir injusticias precedentes. Así, por ejemplo, al político que resulta pospuesto o excluido de una candidatura en la que el partido está obligado a proponer a una mujer y que argumenta que su exclusión o posposición se ha producido sólo por razón de su sexo, Ruiz Miguel le responde que ese reproche es un mero subterfugio que sólo tiende a ocultar que, de no existir la reserva, la preferencia del partido habría sido para un hombre por el mero hecho de serlo, es decir, también por razón de su sexo, consagrando así la desigualdad tradicional en este ámbito. ${ }^{31}$

¿Y si el político postergado está diciendo algo diferente: Que se ha preferido a una mujer por razón de su sexo cuando en realidad él tenía mayores méritos? Probablemente esta es la objeción constitucional más fuerte a la implementación de cualquier sistema de cuotas: que ninguna política pública puede sustentarse en la violación de los derechos fundamentales, aunque sea un mecanismo eficaz para lograr un objetivo socialmente deseable. Luego, aquellos - por ejemplo, los hombres- que podrían quedar en una posición desventajosa para acceder a alguna posición o cargo, y que incluso podrían ser excluidos por la asignación de cuotas para mujeres, serían vulnerados en sus derechos puesto que no son responsables directos de la discriminación que sufren las mujeres.

Para responder vale la pena recordar la réplica de Dworkin a quienes criticaron la decisión de la Universidad de Washington en el caso De Funnis. "Es obvio que De Funnis no tiene derecho constitucional alguno a que (...) la inteligencia sea el criterio exclusivo de admisión (...) los estándares intelectuales se justifican, no porque favorezcan a los inteligentes, sino porque aparentemente sirven a una práctica social útil (...) en ocasiones las facultades de derecho sirven mejor a dicha práctica complementando las pruebas de inteligencia con otro tipo de estándares" 32 incluyendo a minorías raciales o veteranos de guerra.

Dicho de otro modo -y para aplicarlo directamente al caso de las cuotas- el argumento sostiene que ningún hombre puede reclamar que sus derechos son infringidos por una ley de cuotas puesto que no es posible argumentar que merezca a priori (debido a sus mayores "méritos") acceder a un cargo. La idea de mérito en abstracto es equívoca debido a que éste no existe sin referencia a ciertos objetivos institucionales previamente definidos como valiosos, luego, poseer un cierto rasgo o determinadas características políticas no puede constituir nunca un punto de vista independiente desde el cual pueda criticarse un determinado método de selección pues será cada institución la que decida qué es, para el caso concreto, lo que se necesita para acceder al cargo. De este modo, los aspirantes hombres no tienen un derecho absoluto a un cierto cupo electoral en base a méritos o habilidades políticas como, por ejemplo, la facilidad de presentación de

\footnotetext{
${ }^{31}$ Ibid., pp. 259-260.

${ }^{32}$ Dworkin, R., Los derechos en serio, op. cit., p. 330.
} 
problemas y soluciones, la destreza verbal, la capacidad de generar confianza y captar adhesiones, la flexibilidad ante los cambios, la capacidad para entender al electorado, la prudencia en la acción y la gestión, la generación de nuevas ideas, la fidelidad a un ideario, etc. "Pues bien, no parece que cualidades como las anteriores, por lo demás difícilmente objetivables o cuantificables por separado y no necesariamente compatibles y jerarquizables entre sí, se hallen más y mejor distribuidas entre los hombres que entre las mujeres". ${ }^{33}$ Ningún candidato a ningún puesto competitivo tiene un derecho anterior a exigir que un determinado talante que le es especialmente favorable (como las cualidades políticas) sea el que definitivamente determine quién ganará dicho cupo. La cláusula de igual protección ante la ley no excluye -por el contrario, exige algunas veces- la consideración de determinados rasgos (como el sexo o la raza) al momento de adjudicar cargos de representación popular.

En definitiva, las cuotas no afectan derechos básicos sino sólo la distribución de bienes sociales que, en diferentes contextos, autorizan restricciones en función de fines de interés público, como ocurre al fomentarse la contratación de jóvenes, mayores de edad, personas con cierta discapacidad, etc. Al fin y al cabo, ¿estamos seguros que cuando se selecciona a los hombres para asumir los más altos cargos de responsabilidad política, para ser presidentes o consejeros de bancos y grandes empresas, incluyendo los medios de comunicación y las universidades, se hace sólo en base a sus "méritos" profesionales y no, precisamente, debido a que son varones? ¿Realmente pueden quienes hoy manejan más del $90 \%$ de los puestos de mayor relevancia y mejor remunerados en el mundo demostrar que el sexo no ha sido determinante a la hora de calificar sus méritos profesionales? Qué duda cabe que en la selección de ciertos puestos de trabajo se tienen en cuenta calificaciones dentro de las cuales el sexo funciona a modo de "mérito", así como se considera que para pertenecer a un cierto equipo de básquetbol en necesario medir una cierta estatura. ${ }^{34}$

Aún más, incluso dejando de lado que en la política real las designaciones de los candidatos pueden llegar a tener poco que ver con sus méritos, el principio de igualdad exige que en la selección de ciertos puestos no sólo se tengan en cuenta ciertas calificaciones, sino también otros objetivos importantes como la integración o la paz social, o que la raza o el sexo pueden operar en ciertos casos como méritos (o la altura, como en el ejemplo del deportista). Lo decisivo es que no hay argumentos que nos permitan defender la tesis de que las cualidades (méritos) necesarias para ocupar cargos políticos “se hallen más y mejor distribuidas entre los hombres que entre las mujeres. Y si eso es

33 Ruiz Miguel, A., "La representación democrática de las mujeres”, op. cit., p. 260.

34 “¿Podemos sostener que las cualidades que parecen preferibles para el ejercicio de la política se hallan preferentemente en los hombres y no en las mujeres al punto de justificar los actuales grados de participación femenina en política?" Ciertamente no. "Frente a supuestas diferencias esenciales, las cualidades más apropiadas para la política no pasan limpiamente por las categorías de lo masculino y lo femenino. En el peor de los casos, como se ha ironizado alguna vez, las mujeres tienen derecho a actuar en política tan mal como cualquier hombre". Ruiz Miguel, A., "La discriminación inversa y el caso Kalanke”, op. cit., pp. $259-260$. Destacado agregado. 
así, una política de cuotas difícilmente puede afectar de manera sensible a la igualdad de mérito, salvo, naturalmente, que no exista un número suficiente de mujeres con actividad en los partidos, en cuyo caso la propia política de cuotas, gradualmente aplicada, debería servir para llegar a él con rapidez". 35

En conclusión, como cualquier estándar pondrá siempre a ciertos candidatos en desventaja frente a otros, una política de discriminación inversa será perfectamente admitida por el principio de igualdad si parece razonable esperar que el beneficio global para la comunidad exceda a la pérdida global y si ninguna otra política produjera, incluso aproximadamente, el mismo beneficio sin traer aparejada una desventaja comparable. ${ }^{36}$ Como es, según se ha argumentado, el caso de las cuotas electorales que se revisan a continuación. ${ }^{37}$

\section{LOS SISTEMAS DE CUOTAS ELECTORALES}

Los sistemas de cuotas electorales son acciones positivas que parten del supuesto de que existe un desequilibrio que impide la igualdad de oportunidades entre mujeres y hombres. En ese sentido es posible sostener los siguientes argumentos políticodemocráticos a su favor: i) Los derechos políticos de las mujeres implican no sólo su derecho a elegir representantes, sino que también a ser elegidas. En la actualidad, ellas no gozan de este derecho en forma igualitaria; ii) Las mujeres representan más de la mitad de la población mundial, por lo que también debieran tener la mitad de los puestos de representatividad; iii) Las mujeres tienen diferentes experiencias sociales y biológicas, además de diferentes intereses, que deben ser representados en la esfera pública. ${ }^{38}$

Los sistemas de cuotas electorales para mujeres son usados por distintos Estados en todo el mundo en parte debido a la influencia ejercida por diversas recomendaciones de organismos internacionales, así como por la motivación generada por su adopción en otros países. Es sensato predecir que una adecuada participación femenina en la política estatal es muy probable que sirva para alcanzar una mayor igualdad social entre mujeres y hombres. Y ello tanto por la eficacia directa que la legislación y el gobierno

${ }^{35}$ Ruiz Miguel, A., "La representación democrática de las mujeres”, op. cit., p. 260.

36 Dworkin, R., Los derechos en serio, op. cit., p. 333.

${ }^{37}$ Finalmente, no desconocemos el carácter severo y controversial de las cuotas ya que, como se ha señalado, las medidas de discriminación inversa implican la reserva de un cupo en situaciones de especial escasez y, por lo tanto, afectan la distribución de bienes sociales preciados. Por este motivo los sistemas de cuota deben ser esencialmente temporales en su implementación. Ruiz Miguel advierte que "se trata de políticas limitadas que sin duda han de diseñarse y calibrarse con precaución y aplicarse con mesura, seguramente sólo en sectores estratégicos o especialmente importantes y con cierto carácter excepcional y provisional, esto es, en tanto la situación de desigualdad mejora”. Ruiz Miguel, A., "Discriminación inversa e igualdad", op. cit., p. 85.

${ }^{38}$ Ríos, M., op. cit., p 30. 
pueden ejercer sobre las relaciones sociales (en especial en lo que concierne al reparto de los papeles familiares, decisivo para la desigualdad femenina) como también por la influencia indirecta que el desempeño por parte de las mujeres de los puestos de mayor relevancia política puede tener en la transmisión de modelos de rol más igualitarios y en la superación de los estereotipos que dificultan su acceso a los demás puestos de especial relevancia social. ${ }^{39}$

Algunos critican que estas fórmulas electorales "quiebran la libertad electoral" en cuanto que quien es elegido lo hace, no porque los votantes lo deciden, sino porque pertenece a una minoría. Esta objeción, con todo, se refiere a la eventual asignación directa de puestos en el Parlamento para una o varias minorías, al modo de los senadores designados y al margen de todo proceso electoral. Pero las cuotas electorales que de hecho se vienen estableciendo y propugnando para aumentar la representación femenina en los parlamentos no tienen nada que ver con este modelo, pues no reservan directamente un cupo en el órgano parlamentario con independencia de la elección, sino un cupo en las candidaturas electorales, por lo que las personas son elegidas por los votantes. ${ }^{40}$ Luego, esta forma de implementación de cuotas electorales varía desde la adopción voluntaria por los partidos políticos hasta su reconocimiento constitucional, rango en el que se incluyen las leyes de cuotas. Esta última es la forma más usada en América Latina.

Durante la década de los noventa once países latinoamericanos adoptaron leyes para el establecimiento de cuotas que garantizaran a las mujeres niveles mínimos de participación política como candidatas en las elecciones nacionales. Si bien los resultados de esta medida varían dependiendo, entre otros factores, de los sistemas electorales y del apego a las normas por parte de los partidos políticos, es posible sostener que, en términos generales, éstas han sido efectivas. Así, de los 18 países que lideran el ranking de participación femenina en los parlamentos, sólo tres no poseen algún mecanismo (ley de cuotas o cuotas de partido) relacionado con las cuotas. ${ }^{41}$

A continuación se realizará una breve exposición del funcionamiento de las leyes de cuotas en Argentina y Costa Rica, en atención a que en estos países se han observado los resultados más exitosos a partir de los cuales es posible extraer lecciones útiles para el caso chileno. Es necesario señalar que estos casos no son representativos del funcionamiento de este tipo de leyes en toda América Latina sino que se mencionan como paradigmas. ${ }^{42}$

\footnotetext{
${ }^{39}$ Ruiz Miguel, A., "La discriminación inversa y el caso Kalanke”, op. cit., p. 246.

${ }^{40}$ Ibid., p. 248.

41 Ríos y Villar, op. cit., p. 11.

${ }^{42}$ Para un estudio de la experiencia latinoamericana con las cuotas, véase Peschard, J. "El sistema de cuotas en América Latina: Panorama general”, en La aplicación de las cuotas: experiencias latinoamericanas. International Institute for Democracy and Electoral Assistance, IDEA, 2003.
} 


\section{i) El sistema de cuotas en Argentina}

Argentina fue el primer país de Latinoamérica que adoptó, en 1991, un sistema de cuotas para la inclusión de las mujeres en el Congreso. La ley de cupos estableció que las listas de candidatos, propuestas por los partidos políticos, deberían contener al menos un $30 \%$ de candidatas mujeres. Además, dispuso que no sería oficializada ninguna lista que no cumpliera aquel requisito. Antes de la implementación de la ley, el índice de mujeres en el Congreso era de un 3\%, un porcentaje mínimo considerando que las mujeres participaban en un $48 \%$ en los distintos partidos políticos. Desde la entrada en vigencia de la ley, la tasa de participación femenina en el Congreso aumentó considerablemente alcanzando, en el año 2003, un 31,2\% y un 35\% en el año 2005. Sin embargo, la evaluación de las cuotas en el Poder Legislativo no sólo fue favorable en cuanto a números, sino también porque muchas leyes, tales como las de violencia y salud reproductiva, no habrían sido aprobadas si no existieran mujeres comprometidas con estas temáticas en los parlamentos. ${ }^{43,44}$

Otro aspecto fundamental a considerar es que, con posterioridad a la adopción de la ley de cuotas, se verificó una mayor presencia de mujeres, no sólo en la competencia electoral, sino que por medio de un efecto multiplicador en otras organizaciones de la sociedad civil. Por ejemplo, las mujeres sindicalistas se movilizaron a favor de las cuotas en las asociaciones gremiales y mujeres de asociaciones profesionales plantearon reclamos judiciales por su exclusión de las comisiones directivas de sus organizaciones. ${ }^{45}$ Es así como la implementación de una ley de cuotas en Argentina no sólo generó un simple aumento en la cantidad de parlamentarias, sino que su incorporación también en aquellos cargos directivos tradicionalmente ocupados por hombres más allá del ámbito electoral logrando, como sostiene Dworkin, un beneficio global para la comunidad.

\section{ii) El sistema de cuotas en Costa Rica}

En el año 1996 Costa Rica estableció por ley un mecanismo de discriminación inversa, consistente en una cuota mínima de participación femenina en los cargos políticos de elección popular, así como en las estructuras partidarias. Este hito dio cuenta de una nueva etapa en la participación política de la mujer en ese país desde que, en 1949, se

${ }^{43}$ Lubertino, M. J., "Pioneras en las cuotas: la experiencia argentina y su futuro", en La aplicación de las cuotas: experiencias latinoamericanas, IDEA, Estocolmo, 2004, p. 43.

${ }^{44}$ Además, el aumento de mujeres en las cámaras contribuyó significativamente al mayor acceso de ellas a posiciones de liderazgo en las distintas comisiones parlamentarias. Así, en 1991 -año en que se aprobó la ley- el porcentaje de diputadas en tales posiciones fue de un 16,1\%, tasa que aumentó considerablemente en el año 2002 alcanzando un 51,6\%. Mientras que en el caso de las senadoras, desde un 16\% en 1992, se avanzó hasta alcanzar un 31,1\% en el año 2002. Archenti, N., "Los caminos de la inclusión política, acciones afirmativas de género", en Hombres públicos, mujeres públicas, Fundación Friedrich Ebert, Buenos Aires, 2002, p. 43.

${ }^{45}$ Ibid., p. 45. 
reconoció el derecho a voto femenino y la consiguiente opción de ser elegida. Antes de la nueva ley, la proporción de mujeres en la Asamblea Legislativa era menor al 10\%. A partir del establecimiento de políticas para la mujer y de las cuotas en los años noventa, esa proporción pasó a ser de un $19 \%$ en 1998 y un 35\% en el $2002 .{ }^{46}$ En las elecciones del año 2006 alcanzó un 38,6\%.

El proceso de implementación de la ley de cuotas se inició en 1988 con los primeros proyectos de ley que la contemplaban; pero no fue sino hasta el año 1996, mediante una reforma al Código Electoral, que se estableció la cuota mínima de participación femenina. Esta reforma obligaba a los partidos políticos a contener en sus estatutos un mecanismo que asegure la participación de las mujeres en un $40 \%$, tanto en la estructura partidaria como en las papeletas para los puestos de elección popular. Contempló, además, una disposición que señalaba que cuando un partido haya alcanzado la participación política de la mujer en proporción a su número dentro del padrón electoral, cesarán dichas medidas, mediante resolución del Tribunal Supremo de Elecciones. ${ }^{47}$

¿Cuáles han sido los resultados de la inclusión de la mujer en el Parlamento costarricense? Según han señalado estudios realizados por el Boletín Parlamentario de Costa Rica relacionados con la contribución de la mujer en la política, los proyectos de ley sometidos a aprobación de la Asamblea Legislativa por las diputadas han tenido éxito en un $81 \%$ de los casos, mientras que para los hombres la tasa de éxito es sólo de un $48 \%$. La razón sería que las mujeres pasan muchas más horas que los hombres en comisiones parlamentarias y en reuniones con sus electores, específicamente un $70 \%$ de exclusiva participación, frente a un $26 \%$ en los hombres, quienes dedican más tiempo a otras actividades económicas, como su actividad profesional o negocios privados. ${ }^{48}$

Al igual que en el caso argentino, la ley de cuotas en Costa Rica resulta ejemplar tanto por las consecuencias cuantitativas en la representación parlamentaria de las mujeres, como por el efecto extensivo de ésta, que ha propiciado la participación de la mujer en otros ámbitos. Es necesario destacar que, además del resultado directo consistente en la mayor participación de la mujer en los órganos políticos, las cuotas han tenido un importante efecto simbólico, ya que han sensibilizado a la población sobre el problema de inequidad de la mujer. El debate en torno a la adopción de cuotas ha permitido que se vaya creando el hábito de pensar en las mujeres como profesional y políticamente capaces de ocupar cargos de alta responsabilidad. ${ }^{49}$

\footnotetext{
${ }^{46}$ García, A., "Concretando el mandato: reforma jurídica en Costa Rica”, en La aplicación de las cuotas: experiencias latinoamericanas, IDEA, Estocolmo, 2004, p. 103.

${ }^{47}$ Ibid., p. 97.

${ }^{48}$ Olsen, K., "El camino hacia la igualdad. Las mujeres costarricenses en el Parlamento”, en M. MéndezMontalvo y J. Ballington (editoras), Mujeres en el Parlamento: más allá de los números, IDEA, Estocolmo, 2002, p. 151.

${ }^{49}$ Peschard, J., op. cit., p. 28.
} 
iii) ¿Son posibles las cuotas en el sistema binominal chileno?

Diversos estudios muestran que los sistemas de representación proporcional (SRP) son más favorables a la representación femenina que los sistemas mayoritarios. ${ }^{50}$ Así, los 15 países que encabezan la lista de Estados con mayor representación femenina utilizan alguna variante del SRP. ${ }^{51}$ Dentro de estos sistemas, los que permiten un mayor acceso de las mujeres son los que garantizan una elevada magnitud de partido y una magnitud de distrito, de ahí que el sistema binominal resulte tan poco adecuado para el acceso de las chilenas al Parlamento. Si sólo es un candidato el que se postula -baja magnitud del distrito-, lo más probable es que se trate de un hombre pues él es visto como el candidato "más ampliamente aceptado". Este obstáculo es menor si la magnitud del distrito es mayor y pueden postular y ser elegidas varias personas de un mismo partido sin poner en riesgo una plaza "masculina". 52

Como se sabe, el sistema electoral chileno binominal es una especie de sistema proporcional con una baja magnitud de distrito (sólo dos escaños por distrito), lo que implica un fuerte efecto mayoritario. Ello sumado a sus listas abiertas y baja competitividad, hace que las posibilidades de las mujeres de ser representantes en las elecciones resulten escasas. El sistema binominal, luego, ha "magnificado las barreras de entrada y los obstáculos que deben enfrentar las mujeres para acceder al Congreso". 53

Ahora, cuando se trata de cuotas electorales, es necesario tener presente que existen diversos tipos, distinguiéndose principalmente entre "cuotas voluntarias de partido" por un lado y las "cuotas constitucionales y legislativas" por otro. Las cuotas constitucionales están consagradas en la Constitución del país, mientras que las cuotas legislativas se establecen en la ley electoral, la ley de partidos políticos o alguna otra ley comparable. El incumplimiento de las cuotas legislativas o constitucionales puede ocasionar sanciones para aquellas entidades políticas que no las aplicaron. Como ejemplos de sanciones impuestas por las autoridades legales de un país están la descalificación de candidatos, la imposición de multas, e inclusive la descalificación de todo el partido. ${ }^{54}$

Por el contrario, las cuotas voluntarias de partido son fijadas por los propios partidos para garantizar la postulación de un determinado número o proporción de mujeres. Tal como lo indica su nombre, las cuotas voluntarias de partido no son legalmente obligatorias, y por lo tanto no existen sanciones para exigir su cumplimiento. Estas últimas

${ }^{50}$ Véase Unión Europea, Impacto diferencial de los sistemas electorales en la representación política femenina. Dirección General de Estudios. Serie: Derechos de la mujer. 1997; Ríos y Villar, cit.; Peschard, J., cit.; Larserud y Taphorn, Diseño para lograr la igualdad. Combinaciones más idóneas, medianamente idóneas y no favorables de los sistemas electorales y cuotas de género. IDEA, Estocolmo, 2005.

${ }^{51}$ Ríos, M., Cuotas de género: democracia y representación. Santiago, Chile: FLACSO, 2006, p. 5.

52 La magnitud del distrito se encuentra relacionada con la magnitud partidaria, esto es, con el número de candidatos de un partido elegidos en un distrito electoral. La magnitud partidista será mayor si los distritos son grandes y si el número de partidos que ganan las elecciones es relativamente bajo. Larserud y Taphorn, op. cit., p. 11.

${ }^{53}$ Ríos, M., op. cit., p. 5.

${ }^{54}$ Ibid. 
son las que actualmente existen en Chile y los porcentajes de participación femenina varían dependiendo del partido político, aunque en ninguno de ellos se ha superado el $28 \%$ del total de candidatos. ${ }^{55}$ Lo anterior significa que el $90,3 \%$ de los candidatos presentados por los partidos entre 1989 y 2005 han sido varones. ${ }^{56}$

Luego, ¿pueden ser efectivas las cuotas -legales- en el actual sistema binominal? Se ha sostenido que las posibilidades de éxito de un proyecto de ley como el presentado el año 2003 -que exige que en la declaración de candidaturas de cada partido o pacto ningún sexo supere el $60 \%$ del total de candidaturas presentadas- son escasas debido a las restricciones del sistema binominal en términos de magnitud de partidos y de distritos. Como no todos los partidos pueden presentar candidatos, y cuando lo hacen sólo presentan uno (pues solo hay una posición que llenar por partido), es muy difícil aplicar las cuotas para mujeres. ${ }^{57}$ Para evitar este problema se debiera estipular que el equilibrio de sexos se garantice no en las listas de cada partido, sino que en las listas de los pactos y coaliciones de modo que al menos uno de los dos candidatos que se presenten sea mujer.

Aún así, no cabe duda que una reforma más profunda del sistema binominal en el sentido de garantizar en general la representación de las "minorías sociales" sería un mecanismo más efectivo para solucionar la casi total ausencia de mujeres en el Parlamento chileno. Por lo tanto, una clara conclusión es que el sistema electoral y las cuotas que se utilicen deben considerarse en conjunto y no por separado, ${ }^{58}$ es decir, resulta fundamental que la discusión sobre la incorporación de las cuotas en el sistema electoral chileno se realice al mismo tiempo con la discusión relativa a las modificaciones al sistema binominal.

\section{CONCLusión}

Siguiendo el principio de compensación de Rawls puede ser útil, para comprender la relación que existe entre las cuotas electorales y el principio de igualdad de oportunidades,

${ }^{55}$ Desde 1990, los partidos de la coalición gobernante han aprobado medidas voluntarias de cuotas para la elección de sus órganos internos y para los cargos de elección popular. El Partido por la Democracia estableció una cuota del $40 \%$, el Partido Socialista, del $30 \%$ y la Democracia Cristiana, una cuota del 20\%. En la última elección de 2005 el Partido Socialista alcanzó un 27,2\% de participación femenina -promediando un $14,7 \%$ en los últimos quince años. Por su parte, el Partido por la Democracia presentó un 26,6\% de candidatas, aunque promedia sólo un 16,6\%. Respecto al resto de los partidos, considerando sólo la última elección, Renovación Nacional presentó un 16,6\% de candidatas; la Democracia Cristiana un 11,6\%; la Unión Demócrata Independiente un 8\% y finalmente el Partido Radical Social Demócrata que no incluyó mujeres en sus listas.

${ }^{56}$ Es importante tener presente que, si bien las mujeres enfrentan serios obstáculos dentro de sus propios partidos, una vez que logran ser nominadas como candidatas sus expectativas de ser electas son relativamente similares a las de los varones. Ríos, M. op. cit., pp. 29-30.

${ }^{57}$ Ríos, M., “¿Cómo enfrentar la subrepresentación de las mujeres en Chile?”, noviembre 2006, p. 15. En: http://www.fes.cl/documentos/Genero/mrios_progenero.pdf.

${ }^{58}$ Larserud y Taphorn, op. cit., p. 22. 
recordar la clásica imagen de la carrera del economista Lester Thurow, quien sostiene: "Supongamos que se ha identificado a un cierto grupo como víctima de la negación de igualdad de oportunidades, ¿cómo debiera corregirse esta situación? Imaginemos una carrera en la que a algunos corredores se les ha asignado una pesada carga porque pertenecen a un determinado grupo. A causa de esta desventaja el corredor medio con carga quedará rezagado del corredor medio sin carga, aunque algunos corredores con carga adelantarán a algunos corredores sin carga. Ahora supongamos que alguien agita una varita mágica y que las cargas desaparecen de las espaldas de todos los corredores. Si los dos grupos de los corredores son iguales en capacidad, la principal diferencia entre los grupos con carga y sin carga dejará de aumentar, pero aquellos que sufrían por la discriminación previa nunca se equipararán. Más aún, si se tratara de una carrera donde los padres que van primero pudieran pasar el relevo a sus hijos, no habría igualación de la carrera ni siquiera a través de generaciones. La carrera sólo podría volverse limpia si cada uno es obligado a parar y comenzar de nuevo en la misma línea de salida, y si todos los que no llevaban carga fueran obligados a cargar peso hasta que las diferencias en el promedio de ejecución de grupos desaparecieran o si quienes han sufrido desventajas en el pasado, recibieron privilegios especiales hasta que se equiparasen". 59

Corregir las desigualdades entre hombres y mujeres requiere de una equitativa distribución de las funciones sociales, para lo que es imprescindible facilitar y promover la incorporación de la mujer en aquellas áreas en las cuales ha estado tradicionalmente excluida. Considerando la permanente desigualdad de oportunidades que afecta a la mujer en el ámbito público, producto de una cierta definición histórica de los roles sociales, las leyes de cuotas aparecen como un mecanismo de intervención legítimo y capaz de revertir este fenómeno. Para garantizar los mismos derechos de acceder a las vías del progreso no basta con el reconocimiento "formal” a la igualdad pues ello sólo sería efectivo si no existiesen precedentes familiares o, como en el caso de la condición de género, arreglos culturalmente asentados con relación a las diferentes responsabilidades de hombres y mujeres en la sociedad. ${ }^{60}$

Así, como ha defendido John Rawls, para mejorar la situación de los menos aventajados socialmente se requiere instaurar medidas compensatorias de situaciones injustas adscritas, que son las que en la actualidad mantienen a las mujeres lejos del poder. Las cuotas se sustentan precisamente en este principio de compensación que comprende que las condiciones sociales por sí solas no solucionan las desigualdades existentes en una sociedad -como lo muestra el ejemplo de la carrera de Thurow-, por lo que es fundamental que el Estado y demás instituciones sociales básicas (como los partidos políticos) implementen una legislación capaz de generar condiciones de "efectiva igualdad de oportunidades" en el acceso al poder.

59 Thurow, L., "A theory of groups and economics redistribution”, Philosophy and Public Affairs, Vol. 9 $\mathrm{N}^{\circ} 1,1979$, p. 35 .

${ }^{60}$ Fernández, M., "Soy cuotista ¿y qué? Argumentos pro igualdad política de género en un páramo liberal”, en Colección Ideas, Fundación Chile 21, N 66, Santiago, 2006, p. 9. 
Se puede concluir que ni las acciones positivas ni la discriminación inversa vulneran el principio de igualdad, sino que, por el contrario, son una exigencia del mismo principio y están amparados por nuestra Constitución puesto que no implican una diferencia de trato arbitraria. Las cuotas electorales, como mecanismo específico de discriminación inversa, son uno de los medios constitucionalmente admisibles para revertir la actual situación desmejorada de la mujer en la vida pública. Una mayor presencia de las mujeres en el espacio político - tal como lo demuestra la experiencia comparada- contribuirá a profundizar la democracia como aquel espacio público de deliberación ciudadana verdaderamente representativo.

\section{BIBLIOGRAFÍA}

ArChenti, N., "Los caminos de la inclusión política, acciones afirmativas de género", en Hombres públicos, mujeres públicas, Fundación Friedrich Ebert, Buenos Aires, 2002.

BARRÈre, M.A., "Igualdad y discriminación positiva: un esbozo de análisis teórico-conceptual", en Cuadernos Electrónicos de Filosofía del Derecho, No 9, 2003. Disponible en: http://www. uv.es/CEFD/9/barrere1.pdf

Bobbio, N., Igualdad y libertad, Editorial Paidós, Barcelona, 1993.

Bórquez, R.; Ordaz, C., "Participación de las mujeres en organismos internacionales de protección de derechos humanos y en los sistemas judiciales sudamericanos", Domos, año 2, Santiago, 2005.

Dworkin, R., Los derechos en serio, Editorial Ariel, Barcelona, 1984.

- A Matter of Principle, Harvard University Press, 1985.

Fernández, M., "Soy cuotista ¿y qué? Argumentos pro igualdad política de género en un páramo liberal”, en Colección Ideas, Fundación Chile 21, N 66, Santiago, 2006.

García, A., "Concretando el mandato: reforma jurídica en Costa Rica", en La aplicación de las cuotas: experiencias latinoamericanas, IDEA, Estocolmo, 2004.

Kemelmajer, A., "Las acciones positivas", en Miguel Carbonell (compilador), El principio constitucional de igualdad. Lecturas de introducción. Comisión Nacional de los Derechos Humanos de México, México, 2003.

LAPORTA, F., "El principio de igualdad. Introducción a su análisis", Sistema (Revista de Ciencias Sociales) No 67, Madrid, 1985, pp. 3-31.

Larserud y Taphorn, Diseño para lograr la igualdad. Combinaciones más idóneas, medianamente idóneas y no favorables de los sistemas electorales y cuotas de género. IDEA, Estocolmo, 2005.

Lubertino, M. J., "Pioneras en las cuotas: la experiencia argentina y su futuro", en La aplicación de las cuotas: experiencias latinoamericanas, IDEA, Estocolmo, 2004.

Mlynarz, D. y MuÑoz, R., "Mujer: familia, trabajo y participación política. Panorámica de la situación en la última década en Chile”, en Colección Ideas, Fundación Chile 21, N 37 , Santiago, 2003.

Nino, C. S., Introducción al análisis del derecho, Ariel, Barcelona, 2003.

Olsen, K., "El camino hacia la igualdad. Las mujeres costarricenses en el Parlamento", en M. Méndez-Montalvo y J. Ballington (editoras de la edición en español), Mujeres en el Parlamento: más allá de los números, IDEA, Estocolmo, 2002, pp. 147-156.

Peces-Barba, G., Los Valores Superiores, Tecnos. Madrid, 1983. 
Peschard, J., "El sistema de cuotas en América Latina: panorama general", en La aplicación de las cuotas: experiencias latinoamericanas, IDEA, Estocolmo, 2004, pp. 22-31.

RaWls, J., La justicia como equidad. Una reformulación, Ediciones Paidós, Barcelona, 2002.

Rawls, J., A Theory of Justice, Oxford University Press. London, 1971.

Ríos, M., Cuotas de género: democracia y representación. FLACSO, Chile, 2006.

- “Cómo enfrentar la subrepresentación de las mujeres en Chile?”, En: http://www.fes. $\mathrm{cl} /$ documentos/Genero/mrios_progenero.pdf

Ríos, M. y Villar, A., Cuotas de género, democracia y representación, IDEA, FLACSO-Chile, Santiago, 2005.

Ruiz Miguel, A., "Discriminación inversa e igualdad”, en A. Valcárcel (compiladora), El concepto de igualdad, Editorial Pablo Iglesias, Madrid, 1994, p. 80.

_ "La discriminación inversa y el caso Kalanke", Doxa No 19, 1996, pp. 123-140.

- $\quad$ "La representación democrática de las mujeres", Anales de la Cátedra de Francisco Suárez, Universidad de Granada, No 35, 2001, pp. 259-260.

- $\quad$ "La Igualdad en la Jurisprudencia del Tribunal Constitucional”, en El Principio de Igualdad, Luis García San Miguel (ed.), Universidad de Alcalá de Henares-Dykinson, Madrid, España, 2000 .

SChkolnik, M., “¿Por qué es tan increíblemente baja la tasa de participación de las mujeres en Chile?”, en: http://www.expansiva.cl/en_foco/documentos/06102004132738.pdf

ZÚÑIGA, F., "Principio de no discriminación, principio de discriminación compensatoria e igualdad constitucional”, en Anuario de Filosofía Jurídica y Social, Santiago, 2002, pp. 237-260.

\section{Informes $y$ documentos}

Corporación Humanas, Informe Sombra CEDAW, Chile 2003-2006, Estudios y publicaciones, Santiago, 2006.

Corporación la Morada, Mujer-sujeto, mujeres-sujetadas. Informe sobre la situación de los derechos humanos de las mujeres en Chile. Derechos civiles y políticos, Santiago, 2004.

IDEA (Institute for Democracy and Electoral Assistance), Women in Parliament: Beyond Numbers. A Revised Edition, Internacional, IDEA, Estocolmo, 2005.

Servicio Nacional de la Mujer. Informe de seguimiento de la aplicación de la Plataforma de Acción de Beijing presentado por el Gobierno de Chile ante la División para el Adelanto de la Mujer de Naciones Unidas, Santiago, 2004.

Unión EuRopea. Impacto diferencial de los sistemas electorales en la representación política femenina. Dirección General de Estudios. Parlamento Europeo, 1997. 